\title{
Hybrid medical image compression method using quincunx wavelet and geometric actif contour
}

\author{
Haouam Imane', Beladgham Mohammed ${ }^{2}$, Bouida Ahmed ${ }^{3}$ \\ ${ }^{1,2}$ Department of Electrical Engineering, Tahri Mohammed University, Algeria \\ ${ }^{3}$ TIT Laboratory, Tahri Mohammed University of Bechar, Algeria
}

\begin{tabular}{l}
\hline Article Info \\
\hline Article history: \\
Received Jun 30, 2019 \\
Revised Sep 26, 2019 \\
Accepted Oct 24, 2019 \\
\hline
\end{tabular}

\section{Keywords:}

Image compression

Level set (LS)

Medical image

Quincunx wavelet

Set partitioning in hierarchical

Trees (SPIHT)

\begin{abstract}
The purpose of this article is to find an efficient and optimal method of compression by reducing the file size while retaining the information for a good quality processing and to produce credible pathological reports, based on the extraction of the information characteristics contained in medical images. In this article, we proposed a novel medical image compression that combines geometric active contour model and quincunx wavelet transform. In this method it is necessary to localize the region of interest, where we tried to localize all the part that contain the pathological, using the level set for an optimal reduction, then we use the quincunx wavelet coupled with the set partitioning in hierarchical trees (SPIHT) algorithm. After testing several algorithms we noticed that the proposed method gives satisfactory results. The comparison of the experimental results is based on parameters of evaluation.
\end{abstract}

This is an open access article under the CC BY-SA license.

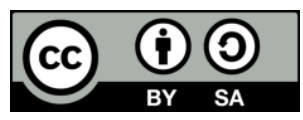

\section{Corresponding Author:}

Haouam Imane,

Department of Electrical Engineering,

Tahri Mohammed university, Algeria.

Email: imahaouam@gmail.com

\section{INTRODUCTION}

Nowdayas, with the technological development, the various applications such as medical imaging require a big space of storage of information or for the transmission, to overcome these constraints, wa velet compression is an inevitable solution. Given the importance of medical images and the many pieces of information they contain, the accuracy of the most important regions and objects in the image is necessary, because it assists an accurate and efficient analysis with credible pathological report with less error. Hence the use of wavelet in a separable way does not allow preserving the outlines and the region of interest, for this purpose the image segmentation is an independent step. The deformable models allows separating image into several regions related with the same properties, and concentrate on specific regions, they are defined as a curve or a contour placed in image and deforms to reach an optimal position. Recently various methods of medical image segmentation have been explored and presented in the literature [1-3] such as level set [4].

The level set (LS) method explained as the operation that aims to gather the pixels of image in regions that constitutes an image partition according to predefined criteria. The idea behind operation is that an initia 1 contour specified by the user is moved to the desired objects boundaries by image driven forces. Where we can specify two forces, while the deformation process started, the internalforces a re designed to conserve the model smooth, where the external forces serve to move the model until the object boundary. The aim of our work is to use an hybrid method, combines the level set with wavelets, for extracting the characteristic information contained in the medical image. Over the years, the wavelets (DWT) have had 
a huge success in image processing field [5]. The idea of wavelet analysis is to decompose a signal on a functional basis of a functional space with well-defined properties [6]. It is to analyze a signal looking for a more compact representation located simultaneously in time and frequency. The wavelet transforms (WT) requires less of calculations on account of their inherently multi-resolution nature, and the encoding schemes which are suited to applications with important scalability and tolera ble degradation

For this paper and including to these two methods and their advantages, we introduce our hybrid method by segmenting the desired object, separate the foreground from the background object before applying a different wavelet transforms coupled with the SPIHT coding. To properly evaluate the compression image quality, we compare the results obtains from various application by the evaluation parameters. Our paper is organised to give, a general introduction, an explanation of level set (LS) method, and quincunx wavelet transform. Finally, the results associate to image quality changes are presented and discussed.

\section{RELATED WORK}

In recent years, the scientific research has shown an increasing interest in the domaine ROI in medicalimage compression, several rea searchers have used many techniques, in order to obtain an optimal compression without losing the quality of the information contain the ROI. In this section, recent research on ROI compression that have:

- In 2015, Manpreet and Vikas [7] divided the image into ROI and Non ROI and used fractal lossy compression for Non ROI image and Context tree weighting lossless for ROI part of an image.

- In 2016, Joshi. p and Rawat. C [8] segmented the image using maximum entropy based thresholding into ROI and Non ROI. After that, they applied lossless compression for ROI using arithmetic coding and lossy compression for Non ROI using SPIHT, this method is evaluated in terms OF PSNR, SSIM.

- In 2017, abdelaziz. E et al [9] introduced a new medical image segmentation approach based on active contour improved by ROI extraction.

- In 2018, C. Priya et al [10] separated the ROI from the Non ROI using fuzzy C means clustering, the Non ROI is compressed using CAVLV method and discret wavelet transform compression method is applied for ROI.

- In 2019, Nafees A et al [11] compresed the image using DWT based zoning technique in combination with DCT for image compression. DWT divides an image into LL, LH, HL and HH frequencies and Zoning is further dividing these images into four parts as an input to DCT one after a nother. The output of DCT on each zone is then combined into a compressed bitstream image.

\section{PROPOSED METHOD}

\subsection{Level set}

Various techniques and methods are designed and applied for image segmentation, are used in the literature [12-13]. It was first introduced by Osher and Sethian [14] who proposed an effective implicit representation for evolving curves and surfaces, it allows for automatic change of topology, such as merging and breaking.

The basic idea for image segmentation is to perform the curves as the zero LS of a higher dimensional hyper-surface. In the LS form, an interface C is depict implicitly as a LS of a function $\varphi$ of higher dimension, which compute the geometric characteristics and the motion of the front. The interface is now represented implicitly as the zero-th LS (or contour). Specific closed curve C, where $C=\partial \omega$, with $\omega \subset \Omega$ being an open subset, for the image $\mathrm{I}(\mathrm{x}, \mathrm{y})$ on the image domain $\Omega$. Either the pixel lies on the curve itself if $\varphi=0$, or it represent the minimum distance between the pixel and the curve.

According to this equation $\frac{\partial c}{\partial t}=F N[15]$ a closed para metric curve is evolved.

Where $\mathrm{t}$ is time, $\mathrm{F}$ is the rate of evolution and $\mathrm{N}$ is the unit normalto the curve. Figure 1 shows. The curve $\mathrm{C}$ evolved according to $\frac{\partial c}{\partial t}=F N$. Figure 2 shows level set segmentation steps. 


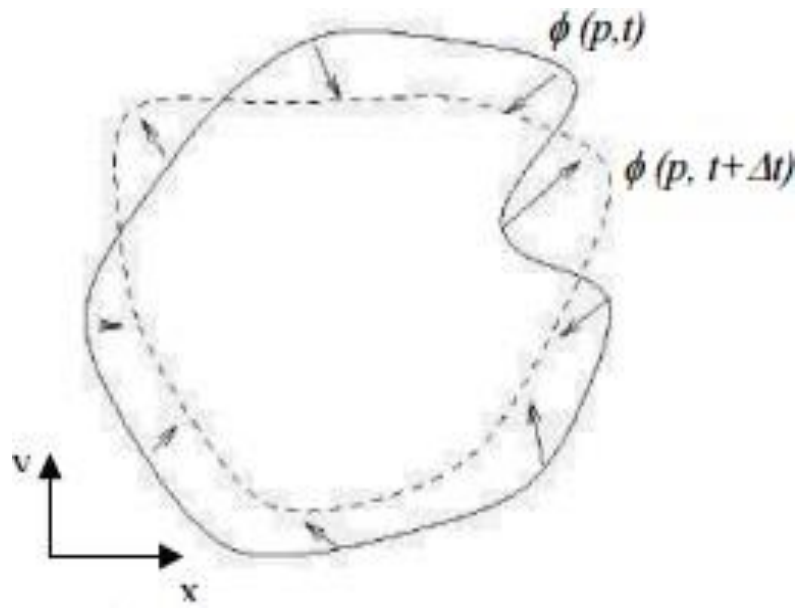

Figure 1. The curve C evolved according to $\frac{\partial c}{\partial t}=F N$

Considering that $\phi(x, y)>0$ if the point (x,y) inside $\mathrm{C}, \phi(x, y)<0$ if the point $(\mathrm{x}, \mathrm{y})$ is outside $\mathrm{C}$ and $\phi(x, y)=0$ if the point (x, y) is on C. Thus, the energy functional F (c1, c2, C) reformulated in terms of the LS function (x, y) as follows [16]:

$$
\begin{aligned}
& F(c 1, c 2, \phi)= \\
& \lambda_{1} \int_{\Omega} I(x, y)-c 1 /^{2} H \varepsilon(\phi(x, y)) d x d y \\
& +\lambda_{2} \int_{\Omega} I I(x, y)-c 2 /^{2}(1-H \varepsilon((\phi(x, y))) d x d y \\
& +\mu \int_{\Omega} \delta(\phi(x, y)) / \nabla \phi(x, y) / d x d y
\end{aligned}
$$

Where $\mathrm{H}(\mathrm{Z})$ and $\delta \varepsilon(z)$ are, respectively, the regularized approximation of Heaviside function $\mathrm{H}$ and delta function $\delta$ as follows:

$$
H(Z)=\left\{\begin{array}{l}
\overrightarrow{1} \text { if }{ }^{-} z \geq 0 \\
0^{-} \text {if } z \prec 0
\end{array} \quad \delta(z)=\frac{d}{d z} H(z)\right.
$$

The LS function $(\mathrm{x}, \mathrm{y})$ is updated by the following gradient descent method:

$$
\frac{\partial \phi}{\partial t}=\delta \varepsilon(\phi)\left[\operatorname{\mu div}\left(\frac{\nabla \phi}{|\nabla \phi|}\right)-\lambda_{1}(I-c 1)^{2}+\lambda_{2}(I-c 2)^{2}\right]
$$

Where $\mathrm{c} 1$ and $\mathrm{c} 2$ are expressed:

$$
\begin{aligned}
c 1(\phi) & =\frac{\int_{\Omega} I(x, y) H \varepsilon(\phi(x, y)) d x d y}{\int_{\Omega} H \varepsilon(\phi(x, y)) d x d y} \\
c 2(\phi) & =\frac{\int_{\Omega} I(x, y)(1-H \varepsilon(\phi(x, y))) d x d y}{\int_{\Omega}(1-H \varepsilon(\phi(x, y))) d x d y}
\end{aligned}
$$




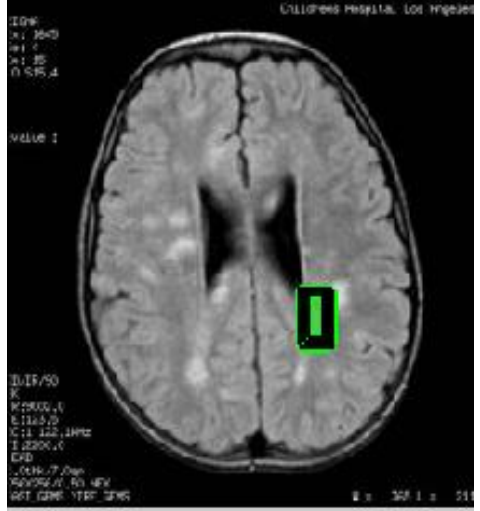

(a)

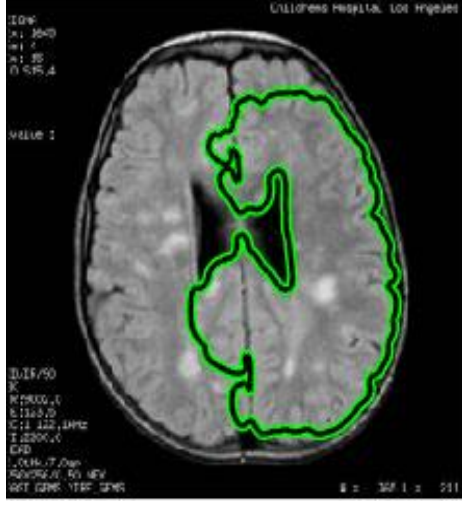

(b)

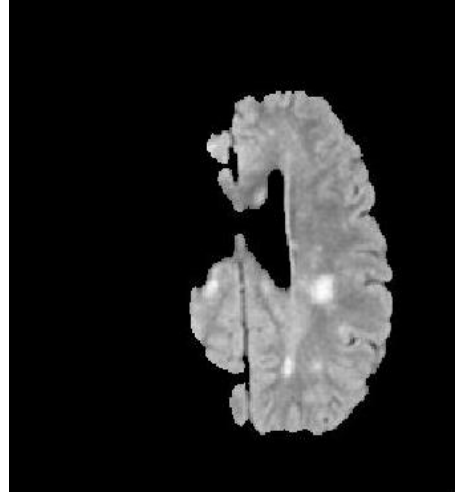

(c)

Figure 2. Level set segmentation steps, (a) Curve initialization, (b) Level set segmentation,

(c) Foreground extraction

\subsection{Quincunx wavelet}

The separable dyadic analysis require three families of wavelets, which is sometimes regarded as a disadvantage, in addition the factor of addition between two successive scales is 4 which may seem high. It is possible to solve these two problems, but at the cost of the loss of filter separability and therefore a slightly higher computational complexity. An analysis has been particularly well studied to find a practical application, known as "quincunx" [17]. Quincunx decomposition results in fewer subbands than most other wavelet decompositions, a feature that may lead to reconstructed images with slightly lower visual quality. The method is not used much in practice, but [18] presents results that suggest that quincunx decomposition performs extremely well and may be the best performer in many practical situations. Figure 3 illustrates this type of decomposition. We notice that the dilation factor is not more than 2 between two successive resolutions, and that only one wavelet family is necessary $[19,20]$.

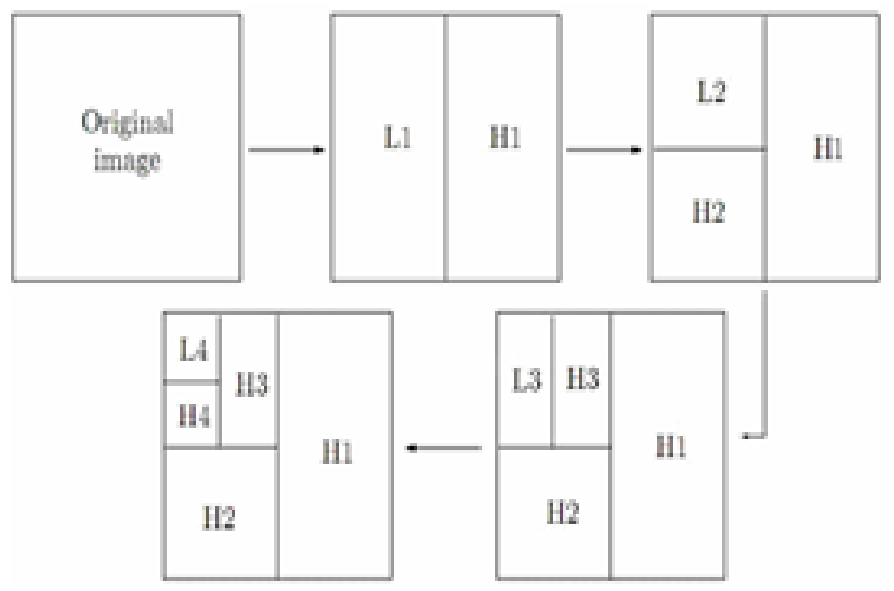

Figure 3. Quincunx wavelet decomposition

In this case the Dilatation matrice will be;

$$
M=\left[\begin{array}{cc}
1 & 1 \\
1 & -1
\end{array}\right]
$$


The Grid transformation (lattice) is done according to $y_{i+1}[n]$ the following diagram:

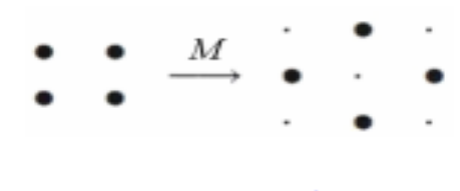

This matrix generates a quincunx lattice in 2D. The column vectors of this matrix form a basis to this lattice. The volume of the unit cell associated equals 2. The same lattice Figure 4 is also emanating from the matrix below [17]:

$$
M^{\prime}=\left[\begin{array}{cc}
1 & -1 \\
1 & 1
\end{array}\right]
$$

It is noticed that the dilatation step is $\sqrt{2}$ on each direction and the geometry of the grid obtained justifies the name given to this multiresolution analysis.

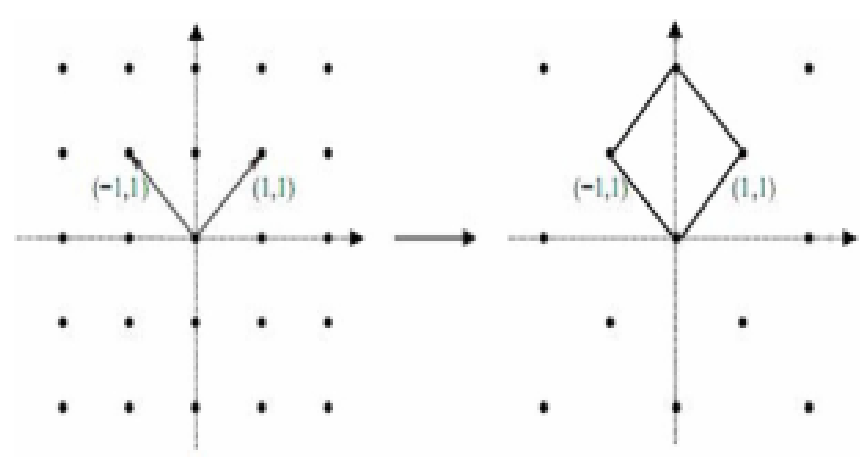

(a)

(b)

Figure 4. Examples of a lattice quincunx and unit cell, (a) Lattice quincunx, (b) Unit cell

\subsubsection{Quincunx sampling and filter}

First, we recall some basic results on quincunx sampling and perfect reconstruction filterbanks $[21,22]$. The quincunx sampling lattice is shown in Figure 4. Figure 5(a) shows Quincunx lattice and Figure 5(b) shows the corresponding Nyquist area in the frequency domain Let $\mathrm{x}[\vec{n}]$ with $\vec{n}=\left(\mathrm{n}_{1}, \mathrm{n}_{2}\right) \in \mathrm{Z}^{2}$ denote the discrete signal on the initial grid. The two-dimensional (2-D) z-transform of $\mathrm{x}[\vec{n}]$ is denoted by:

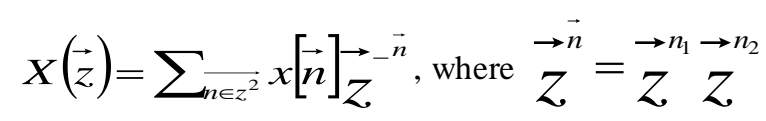

The continuous 2-D Fourier transform is then given by $\left.X\left(e^{j \varpi}\right)=\sum_{n \in z^{2}} x \mid \vec{n}\right\rfloor e^{-j(\vec{w}, \vec{n})}$ with

$$
\overrightarrow{w=\left(w_{1}, w_{2}\right)}
$$

and finally, the discret 2-D Fourier transform for $\mathrm{x}\left[{ }^{n}\right]$ given on an $\mathrm{N} x \mathrm{~N}$ grid $(\mathrm{n} 1, \mathrm{n} 2=0,1, \ldots, \mathrm{N}-1)$ by 


$$
X(\vec{k})=\sum_{\vec{n} \in z^{2}} x|\vec{n}| e^{-j 2 \pi(\vec{k}, \vec{n}) / N}
$$

With $(\mathrm{k} 1, \mathrm{k} 2=0,1 \mathrm{~N}-1)$. Now, we write the quincunx sampled version of $\mathrm{x}\left[\vec{n}_{]}\right.$as:

$$
\left.[x]_{\downarrow M} \mid \vec{n}\right\rfloor=x\lfloor M \vec{n}\rfloor \text { where } M=\left(\begin{array}{cc}
1 & 1 \\
1 & -1
\end{array}\right)
$$

Our down-sampling matrix $\mathrm{M}$ is such that $\mathrm{M}^{2}=2 \mathrm{I}$. where $\mathrm{I}$ is identity matrix. The Fourier domain version of (2) is;

$$
[x]_{\downarrow M}[\vec{n}]=\leftrightarrow \frac{1}{2}\left[X\left(e^{j M^{-T} \vec{\omega}}\right)+X\left(e^{j\left(M^{-T} \overrightarrow{\omega+\vec{\pi}}\right)}\right)\right]
$$

Where $\vec{\pi}=(\pi, \pi)$, The up-sampling is defined by;

$$
[x]_{\uparrow M}[\vec{n}]=\left\{\begin{array}{c}
\left.x \mid M^{-1} \vec{n}\right\rfloor_{, \text {whenn }}+n_{2} \\
\text { Oelsewhere }
\end{array}\right.
$$

and its effect in the Fourier domain is as follows:

$$
[x]_{\uparrow_{M}}|\vec{n}|=\leftrightarrow X\left(e^{j M^{T} \vec{\omega}}\right)
$$

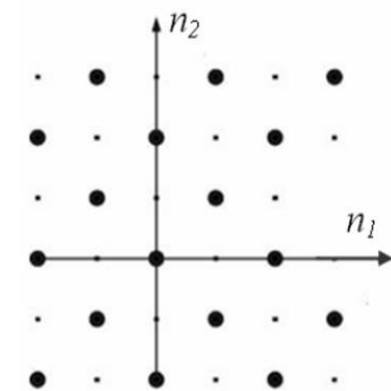

(a)

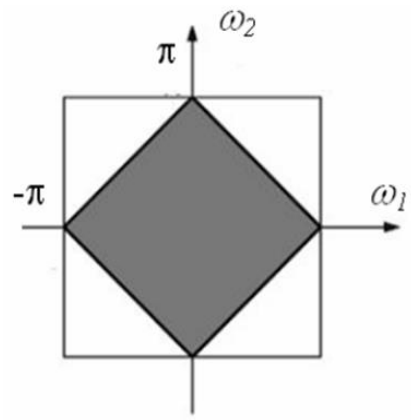

(b)

Figure 5. (a) Quincunx lattice, (b) The corresponding Nyquist a rea in the frequency domain 
If we now chain the down-sampling and up-sampling operators, we get

$$
\begin{aligned}
& {[x]_{\downarrow_{M} \uparrow}[\vec{n}]=\left\{\begin{array}{c}
x \mid \vec{n}\rfloor, \text { whenn }+n_{2} \text { isever } \\
\text { Oelsewhere }
\end{array}\right.} \\
& {[x]_{\downarrow_{M \uparrow M}}[\vec{n}]=\frac{1}{2}\left[X\left(e^{j \vec{\omega}}\right)+X\left(e^{j(\vec{\omega}+\vec{\pi})}\right]\right.}
\end{aligned}
$$

Since quincunx sampling reduces the number of image samples by a factor of two, the corresponding reconstruction filterbank has two channels Figure 6 . The low-pass filter $\tilde{H}$ reduces the resolution by a factor of $\sqrt{2}$; the wavelet coefficients correspond to the output of the high-pass filter $\widetilde{G}[20-22]$.

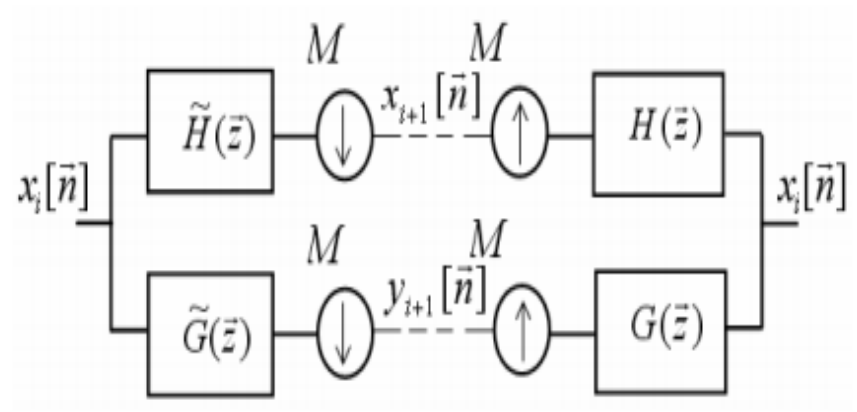

Figure 6. Perfect reconstruction filter bank on a quincunx lattice

\subsubsection{Fractional quincunx filters}

As starting point for our construction, we introduce a new 1-D family of orthogonalfilters;

$$
H_{a}(\omega)=\frac{\sqrt{2}(2+2 \cos \omega)^{\frac{a}{2}}}{\sqrt{(2+2 \cos \omega)^{a}+(2-2 \cos \omega)^{a}}}
$$

This is indexed by the continuously-varying order parameter $\alpha$. Applying the diamond McClellan transform to the filter above is straightforward; it a mounts to replacing $\cos \omega$ by $12(1 / 2)\left(\cos \omega_{1}+\cos \omega_{2}\right)$ in (13). Thus, our quincunx refinement filter is given by [21-22];

$$
H_{a}\left(e^{j \omega}\right)=\frac{\sqrt{2}\left(2+\cos \omega_{1}+\cos \omega_{2}\right)^{\frac{a}{2}}}{\sqrt{\left(2+\cos \omega_{1}+\cos \omega_{2}\right)^{a}+\left(2-\cos \omega_{1}-\cos \omega_{2}\right)^{a}}}
$$

This filter is guaranteed to be orthogonal because the McClellan transform has the property of preserving biorthogonality. Also, by construction, the $\alpha$ th order zero at $\omega=\pi$ gets mapped into a corresponding zero at $\left(\omega_{1}, \omega_{2}\right)=(\pi, \pi)$; this is precisely the condition that is required to get a 2-D wavelet transform of order $\alpha$. Also, note the isotropic behavior and the flatness of $\boldsymbol{H}_{a}\left(\boldsymbol{e}^{j \omega}\right)$ around the origin; i.e, $H_{a}\left(e^{j \omega}\right) / \sqrt{2}=1+O\left(\|\vec{\omega}\|^{a}\right)$ for $\vec{\omega} \rightarrow 0$. The orthogonalwavelet filter is obtained by modulation

$$
G_{a}(\vec{z})=z_{1} H_{a}\left(-\vec{z}^{-1}\right)
$$


The corresponding orthogonal scaling function is defined implicitly as the solution of the quincunx two -scale relation;

$$
\varphi_{a}(\vec{x})=\sqrt{2} \sum_{n \in z^{2}} h_{a}|\vec{n}| \varphi_{a}(M \vec{x}-\vec{n})
$$

Since the refinement filter is orthogonal with respect to the quincunx lattice, it follows that $\varphi_{a}(\vec{x}) \in L_{2}\left(R^{2}\right)$ the partition of unity con $\left(\omega_{1}, \omega_{2}\right)=(\pi, \pi)$ Thus, we have the guarantee that our scheme will yield orthogonal wavelet bases of $L_{2}\left(R^{2}\right)$. The underlying orthogonalquincunx wavelet is simply [23];

$$
\left.\psi_{a}(\vec{x})=\sqrt{2} \sum_{n \in z^{2}} g_{a} \mid \vec{n}\right\rfloor \rho_{a}(M \vec{x}-\vec{n})
$$

\subsection{Spiht coding sheme}

SPIHT algorithm was propound by A. Said and Pearlman [24]. SPIHT performs a recursive partitioning to determine the position of the significant coefficients in the progeny of the considered coefficient. The basic principles of the SPIHT algorithm are a partial storage by amplitude of the wavelet coefficients, then a partitioning in hierarchical at each threshold applied, the trees are sorted on the basis of their meaning in two categories of trees and a scheduling of the transmission of the refinement bits. The following sets of coordinates are used to present the new coding method:

- $\quad O(i, j)$; set of coordinates of all offspring of node $(i, j)$.

- $\quad D(i, j)$; set of coordinates of all descendants of the node $(i, j)$.

- $\quad H$; set of coordinates of all spatial orientation tree roots (nodes in the highest pyramid level).

- $\quad \mathrm{L}(\mathrm{i}, \mathrm{j})=\mathrm{D}(\mathrm{i}, \mathrm{j})-\mathrm{O}(\mathrm{i}, \mathrm{j})$; their algorithm stores the significant information in three ordered lists, to perform a nested coding, called insignificant sets list (LIS), insignificant pixels list (LIP), and significant pixels list (LSP), each entry is signified by (i, j) [23].

The coefficients insignificants in LIP are tested one more time to place those are significant in LSP, in the LIS, the coefficients that will be processed in the next refinement pass are found in the LSP list [25]. Figure 7 shows parent-child relationship.

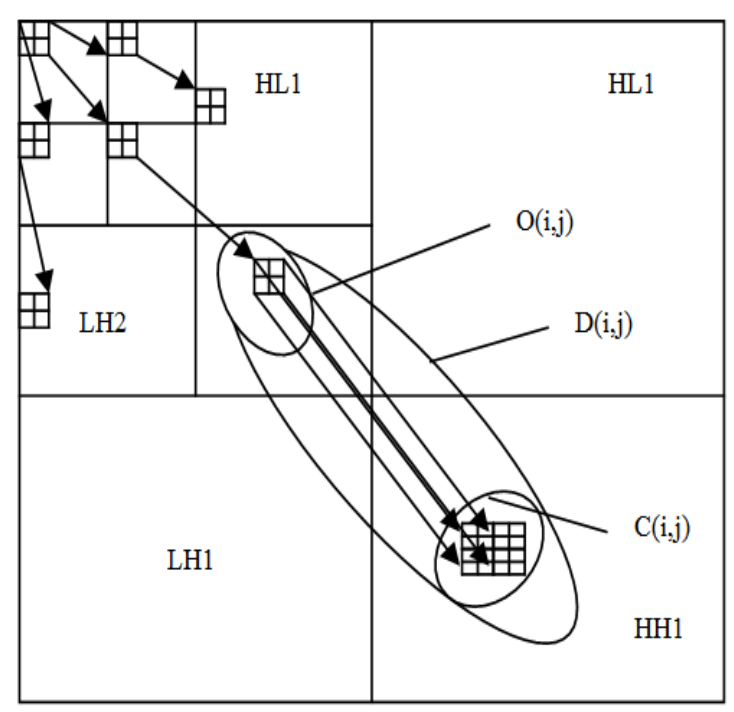

Figure 7. Parent-child relationship

\section{RESEARCH METHOD}

In image compression, minimize the size, keeping the interesting part and showing a good precision of the pathology remains an unresolved problem. This proposed method has been tested on several medical images to see its performance. According to LS method advantages, first of all, we focus on pathological 
images, we segment the object of interest or the part that contains the disease, then after extracting the ROI, the different wavelets are applied on the new image without losing their quality of the image. Based on evaluation parameters such as PSNR and MSSIM, a comparison is given to determine the most powerful algorithm proposed. Figure 8 shows the proposed method steps.

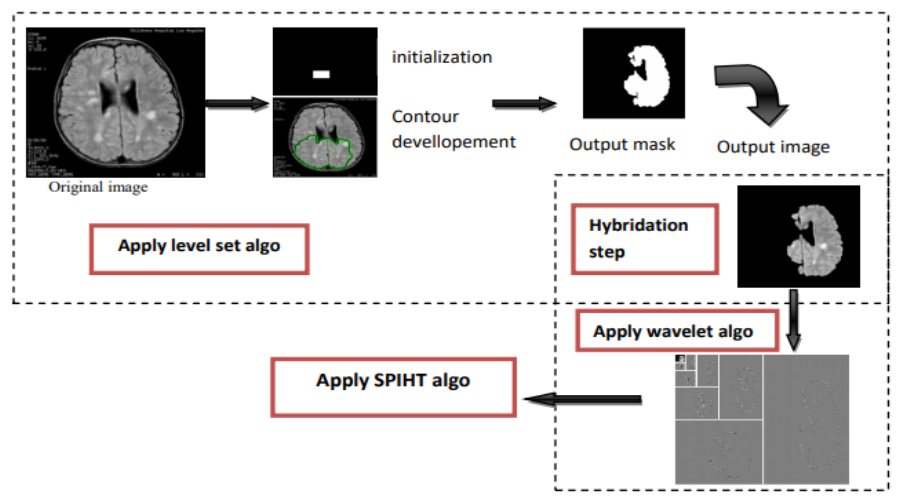

Figure 8. The proposed method steps

\subsection{Quality evaluation parameter}

\subsubsection{The peak signal to noise ratio (PSNR)}

The peak signal to noise ratio (PSNR) used to measure the quality of reconstruction in image compression. It refers to the ratio between signal and reconstruction error variance in Decibel scale. It can be represented as [26]:

$$
M S E=\frac{1}{M x N} \sum_{1=1}^{i=M} \sum_{j=1}^{j=N}(I(i, j)-\hat{I}(i, j))^{2}
$$

Where, MSE: mean squar error between two images. Mean Square Error (MSE) which requires two MxN grayscale images I and $\hat{I}$ where one of the images is considered as a compression of the other. The PSNR is defined as:

$$
P S N R=10 \log _{10}\left(\frac{(\text { Dynamics of } \text { image })^{2}}{M S E}\right)
$$

\subsubsection{The structural similarity index (SSIM)}

This parameter compares the similarity the brightness, contrast and structure between each pair of vectors, the structural similarity index between two signals $\mathrm{x}$ and $\mathrm{y}$ is given by the following expression [26, 27]:

$$
\operatorname{SSIM}(x, y)=l(x, y) \cdot c(x, y) s(x, y)
$$

The MSSIM is used to measure the quality of the local image, which provides more information on the degradation of image quality, which is useful in medical imaging applications. It values exhibit greater consistency with the visual quality.

$$
\operatorname{MSSIM}(I, \hat{I})=\frac{1}{M} \sum_{i=1}^{M} \operatorname{SSIM}\left(I_{i}, \hat{I}_{i}\right)
$$

Where I and $\hat{I}$ are respectively the reference and degraded images, $\mathrm{I}_{\mathrm{i}}$ and $\hat{\mathrm{I}}_{\mathrm{i}}$ are the contents of images at the i-th local window; M: the total number of local windows in image. Finally the quality measurement can provide a spatial map of the local image quality, which provides more information on the image quality degradation, which is useful in medical imaging applications. 


\section{RESULTS AND ANALYSIS}

The aim of our work lies in the possibility of reducing the rates, for which the image quality re mains acceptable, which we are interested in hybrid compression methods based on activ contour segmentation and 2D wavelet transforms because of their interesting properties, applied to medical images. For this reasons the PSNR evaluation parameters and the MSSIM similarity index are used to estimate and judge the quality of the compressed image. In order to verify the efficiency of our hybrid algorithm, we applied, (level set +CDF 9/7 (lifting scheme) + SPIHT) and (level set +quincunx + SPIHT) on MRI medical images of size $512 \times 512$ encoded by 8 bits per pixel. This image is taken from the GE Medical System database [28]. Figure 9 illustrates the steps of the compressed image (region of interest image) by the quincunx wa velet.

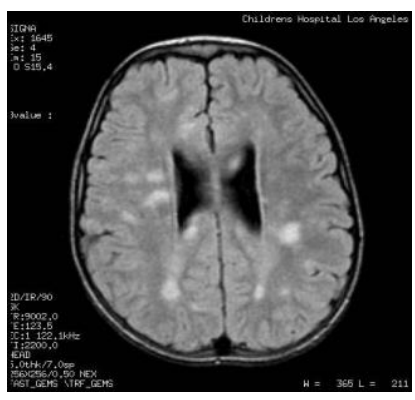

(a)

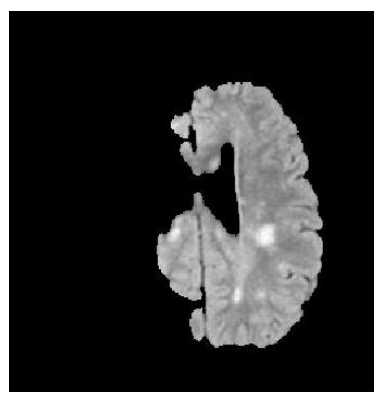

(b)

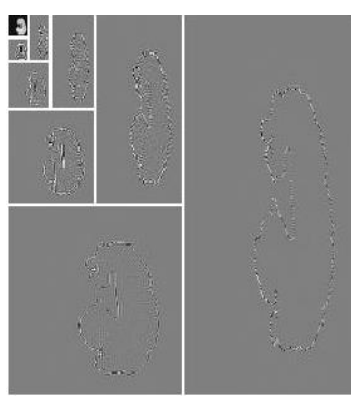

(c)

Figure 9. Image compressionsteps, (a) Brain original image, (b) The region of interest, (c) Quincunx wavelet decomposition

The compressed image quality for different bit-rate values (number of bits per pixel) by the proposed algorithm is presented in the Figure 10. From the results of the PSNR, MSSIM and MSE values obtained, we note that from $0.5 \mathrm{bpp}$, the reconstructed image becomes almost perfect.

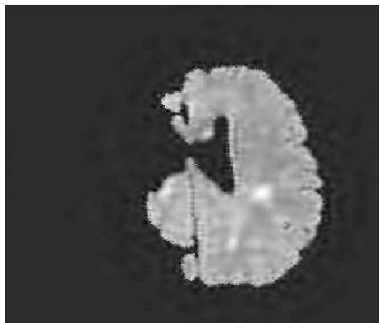

$\mathrm{Rc}=0.25 \mathrm{PSNR}=30.37$ $\mathrm{MSSIM}=0.7$

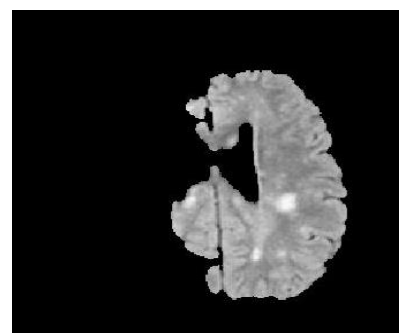

$\mathrm{Rc}=1 \mathrm{PSNR}=48.09$ MSSIM=0.99

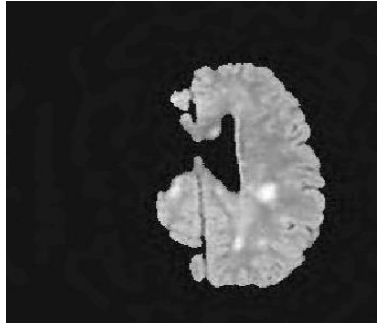

$\mathrm{Rc}=0.5 \mathrm{PSNR}=38.09$ $\mathrm{MSSIM}=0.9$

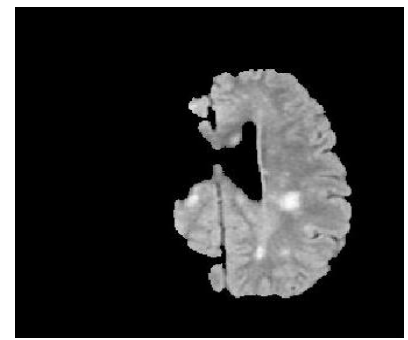

$\mathrm{Rc}=1.5 \mathrm{PSNR}=57.22 \mathrm{MSSIM}=1$

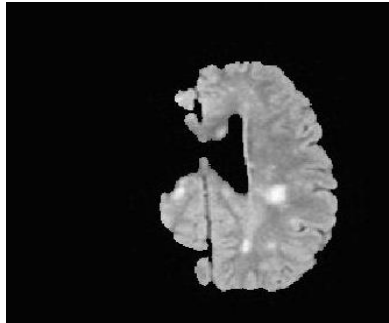

$\mathrm{Rc}=0.75 \mathrm{PSNR}=44.13$ MSSIM $=0.97$

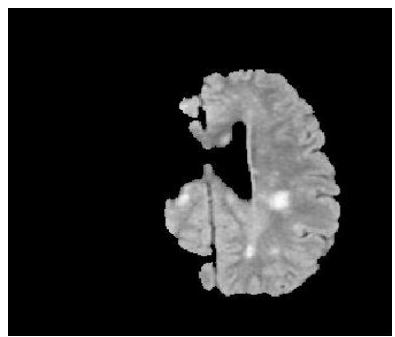

$\mathrm{Rc}=2$ PSNR=66.32 $\mathrm{MSSIM}=1$

Figure 10. Compressing of a ROI of an axial slice with level set coupled with quincunx wavelet and SPIHT coding 
To ensure the performance of our proposed method, we compared between these different types of transform CDF9/7 (Lifting scheme)) coupled with level set method and SPIHT coding and CDF9/7 (Lifting scheme)) coupled with SPIHT coding without applied the level set segmentation applied to the same medical image. The Table 1 and Table 2 given represent respectively these results.

Table 1. PSNR and MSSIM variation using different methods proposed

\begin{tabular}{cccccccc}
\hline \multirow{2}{*}{ Type d'image } & \multirow{2}{*}{ RC (bpp) } & \multicolumn{2}{c}{ Level set +quincun + SPIHT } & \multicolumn{3}{c}{ Level set + CDF9/7 (lifting) + SPIHT } \\
\cline { 3 - 8 } & & PSNR & MSSIM & MSE & PSNR & MSSIM & MSE \\
\hline \multirow{4}{*}{ IRM } & 0.25 & 30.37 & 0.78 & 55 & 29.8 & 0.75 & 16.03 \\
& 0.5 & 38.09 & 0.9 & 10.09 & 36.14 & 0.83 & 7.01 \\
& 0.75 & 44.13 & 0.97 & 2.51 & 42.06 & 0.91 & 2.5 \\
& 1 & 48.99 & 0.99 & 0.82 & 46.55 & 0.96 & 0.79 \\
& 1.5 & 57.22 & 1 & 0.12 & 57.26 & 0,99 & 0.11 \\
& 2 & 66.32 & 1 & 0.02 & 65.09 & 0.99 & 0.05 \\
\hline
\end{tabular}

Table 2. PSNR and MSSIM variation using different methods proposed

\begin{tabular}{ccccccc}
\hline \multirow{2}{*}{ RC (bpp) } & \multicolumn{3}{c}{ Quincunx +SPIHT } & \multicolumn{3}{c}{ CDF9/7 (lifting)+ SPIHT } \\
\cline { 2 - 7 } & PSNR & MSSIM & MSE & PSNR & MSSIM & MSE \\
\hline 0.25 & 38.98 & 0.91 & 8.23 & 36.35 & 0.64 & 15.22 \\
0.5 & 44.33 & 0.96 & 2.4 & 41.51 & 0.79 & 4.63 \\
0.75 & 47.91 & 0.98 & 1.06 & 45.88 & 0.94 & 1.7 \\
1 & 50.75 & 0.99 & 0.56 & 48.15 & 0.96 & 1.01 \\
1.5 & 54.12 & 0.99 & 0.26 & 53.04 & 0.99 & 0.33 \\
2 & 56.45 & 0.99 & 0.16 & 55.80 & 0.99 & 0.18 \\
\hline
\end{tabular}

Comparing the different values of PSNR, MSSIM, and MSE we ensure the efficiency of our algorithm in terms of compressed image quality for the low bit-rate, The Figure 11 shows represent the results of the comparison obtained after applied the different algorithms proposed on an axial slice of brain imaging, given by the PSNR curve.
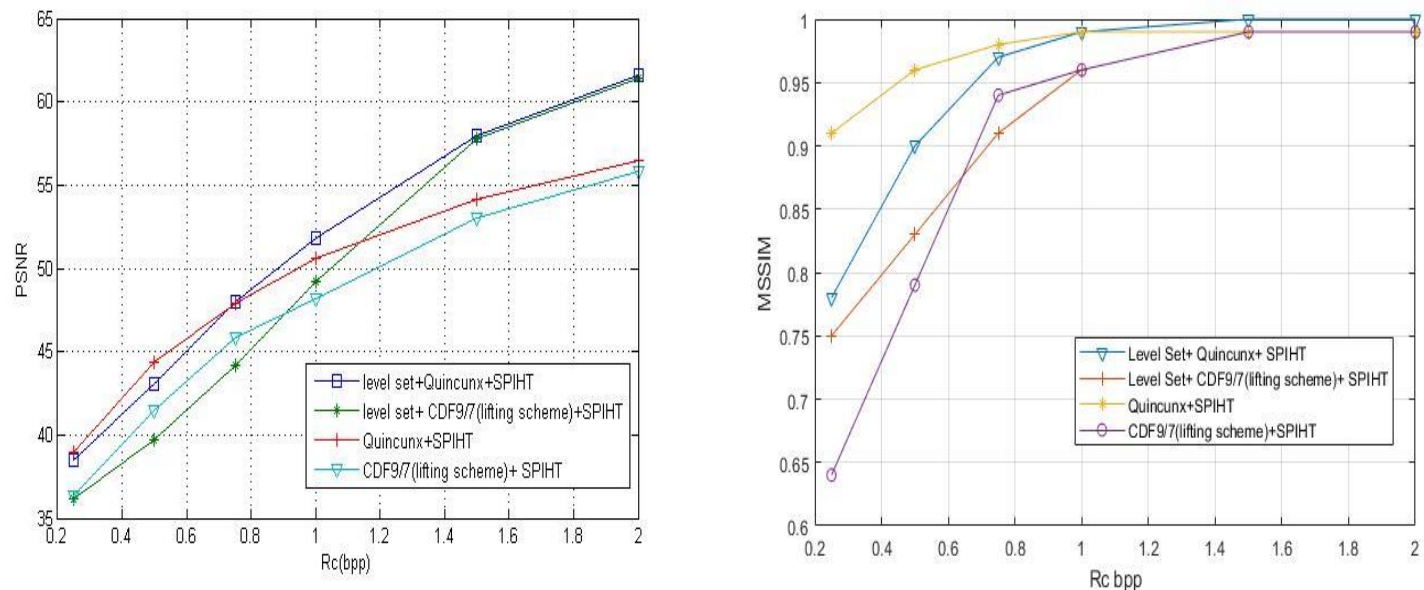

Figure 11. PSNR and MSSIM variation using different proposed methods

The set of medical images in the following figures are used to ensure the performance of the proposed algorithm. For several images and from $0.5 \mathrm{bpp}$ our compression algorithm preserves the image structure and the pathologie form. It's clearly shown the part, the type and the size of pathologie in all tested images. Figure 12 shows ultra sound imaging. Figure 13 shows sagittalsection of brain. Figure 14 shows orthopedic imaging. 


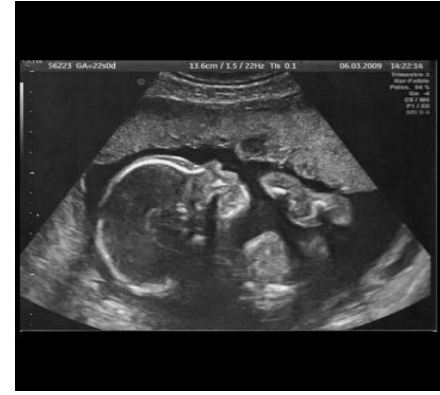

Original image

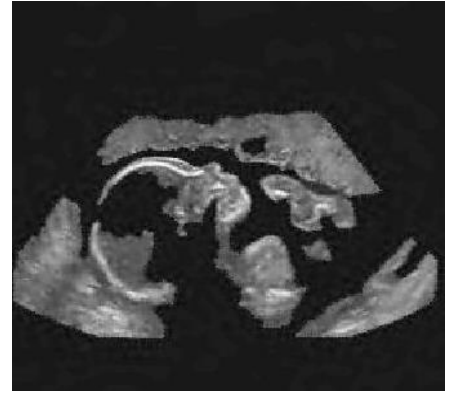

$$
\begin{gathered}
\text { PSNR=36.5, MSE }=14.57 \\
\text { MSSIM=0.93 } \\
\text { Quincunx+LS+SPIHT }
\end{gathered}
$$

Figure 12. Ultra sound imaging

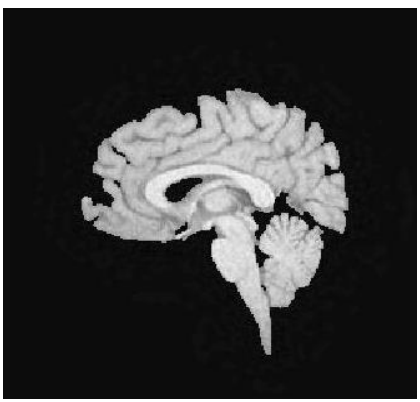

$$
\begin{gathered}
\text { PSNR=47.02, MSE }=1.29, \\
\text { MSSIM=0.99 } \\
\text { Quincun }+ \text { LS+SPIHT }
\end{gathered}
$$

Figure 13. Sagittal section of brain

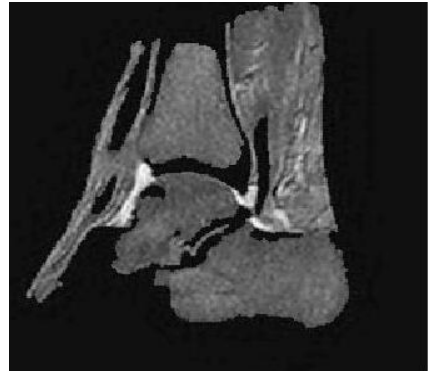

$$
\begin{gathered}
\text { PSNR=37.47, MSE }=11.65, \\
\text { MSSIM=0.94 } \\
\text { Quincunx+LS+SPIHT }
\end{gathered}
$$

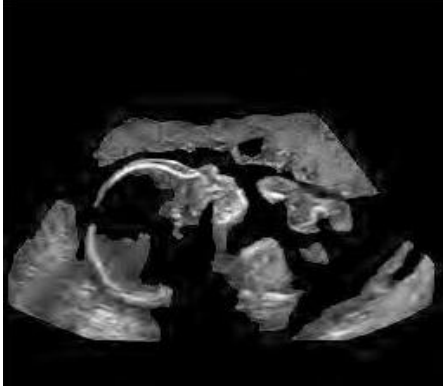

$$
\begin{gathered}
\text { PSNR=33.12, MSE }=31.7 \\
\text { MSSIM }=0.78 \\
\text { CDF9 } / 7+\mathrm{LS}+\text { SPIHT }
\end{gathered}
$$

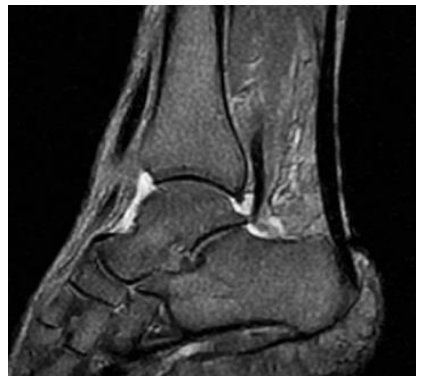

Original image

Figure 14. Orthopedic imaging

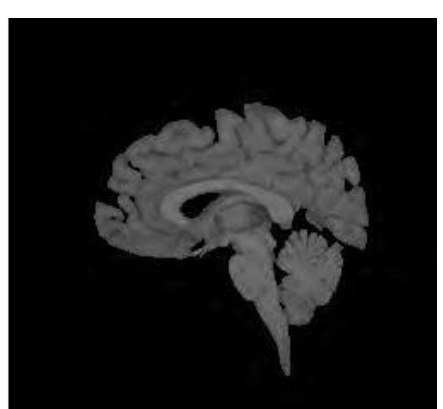
$\mathrm{PSNR}=38.8, \mathrm{MSE}=8.57$, MSSIM $=0.89$ CDF9/7+LS+SPIHT

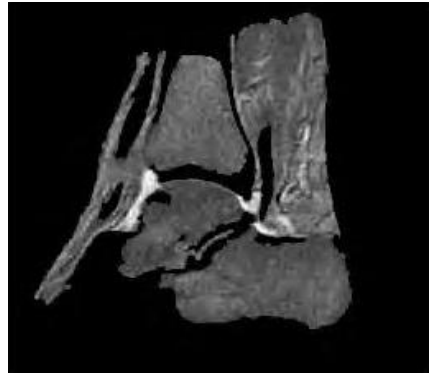
$\mathrm{PNR}=33.65, \mathrm{MSE}=30.63$, MSSIM $=0.79$ CDF9/7+LS+SPIHT

According to the different results obtained for the several application of our hybrid method on color MRI medical image, we ensure that our proposed algorithm gives better quality and it is an efficient method compared with other techniques, more precisely when compressed the region of interest image afterapplying the LS segmentation with quincunx algorithm coupled with SPIHT coding, which gives a better performance and image quality-chapters. 


\section{CONCLUSION}

The work in this paper is to enhance medical images quality after the compression step. We used an hybrid method composed of the LS method and the quincunx wavelet transform applied on variety of medical images. We compared the founding results with the level set coupled with CDF9/7 lifting algo rithm and SPIHT, we notice that the proposed algorithm gives better results than the other compression techniques. Generally the results obtained are very satisfactory in terms of compression ratio and compressed image quality.

\section{REFERENCES}

[1] S. N. Kumar, A Lenin Fred, and S Muthukumar, "A voyage on medical image segmentation algorithms," biomedical research, Special Section: Computational Life Sciences and Smarter Technological Advancement, pp. s75-s87, 2018.

[2] Z. Sanping, W. Jinjun, Z. Shun, "Active Contour Model Based on Local and Global Intensity Information for Medical Image Segmentation," Preprint submitted to Journal of Neurocomputing, vol. 186, pp. 107-118, 19 April 2016.

[3] B. Adegoke, B. Olawale., N. I. Olabisi, "Overview of Medical Image Segmentation," International Journal of Engineering Research and Development, vol. 8, no. 9, pp. 13-17, September 2013.

[4] R. Malladi, J. A. Sethian and B. C. Vemuri, "Shape modeling with front propagation: a level set approach," in IEEE Transactions on Pattern Analysis and Machine Intelligence, vol. 17, no. 2, pp. 158-175, Feb. 1995.

[5] R. N. Ramakant Parida, Swapnil Singh, Chittaranjan Pradhan," Chapter 9 Analysis of Color Image Encryption Using Multidimensional Bogdanov Map,” IGI Global, 2019

[6] H. Soni, C. Modi, H. Shah and R Kher, "Comparative study of different quantization techniques for natural and medical image compression," Proceeding of 2009 International conference on signals, systems and automation (2010), Boca Raton, Florida. 2010.

[7] M. Kaur. and V. Wasson, "ROI Based Medical Image Compression for Telemedicine Application," 4thInternational Conference on Eco-friendly Computing and Communication Systems, ICECCS 2015, Procedia Computer Science, vol. 70, pp 579-585, 2015

[8] Preeti V. J. and Rawat. C. D, "Region Based Hybrid Compression for Medical Images," International conference on Signal Processing, Communication, Power and Embedded System (SCOPES)-2016, pp. 1212-1217, 2016.

[9] E. Abdelaziz, O. E. and B. Abdenbi, "Medical Image Segmentation by Active Contour Improvement," Software Engineering and Applications journal, vol 6, no. 2, pp. 13-17, 2017.

[10] Priya C. and Ramya C, "Medical Image Compression Based On Fuzzy Segmentation", International Journal of Pure and Applied Mathematics, vol. 118, no. 20, pp. 603-610, 2018.

[11] Nafees A et al, "Zone Based Lossy Image Compression Using Discrete Wavelet and Discrete Cosine Transformations," Security and Privacy in New Computing Environments, Second EAI International Conference, SPNCE, Tianjin, China, pp. 731-742, April 2019.

[12] S. Zhou, J. Wang, S. Zhang, and Y. Gong, "Active Contour Model based on Local \& Global Intensity Information for Medical Image Segmentation,” Neurocomputing, vol. 186, pp 107-118, 2016.

[13] P. Nagaswara Reddy and C. P. V. N. J. Mohan Rao, "Brain Mr image segmentation by modified active contours and contourlet transform," ICTACT Journal on image and video processing, 8, November 2017.

[14] J. A. Sethian, "Level set methods and fast marching methods", second ed., Cambridge University Press, 1999.

[15] M. Beladgham, F. Derraz, "Segmentation d'images médicales IRM par la méthode d'ensembles de niveaux (Level_Sets)," Abou- Bekr Belkaid university -Tlemcen, January, 2005.

[16] Shuo Li. et al, "Semi-automatic computer aided lesion detection in dental x-rays using variational level set," Pattern Recognition, vol. 40, no. 10, pp. 2861-2873, 2007.

[17] Chappelier V., "Progressive Coding Of Images By Directed Wavelet", Phd. Thesis, Rennes1 University, December 15 th ,2005.

[18] Stromme, Oyvind, "On the applicability of wavelet transforms to image and video compression," Ph. D. thesis, University of Strathclyde, February 1999.

[19] Truchetet F, "Wavelets for digital signal," Hermes Edition, Paris, January 1998.

[20] Y. Tanaka, M. Ikehara and T. Q. Nguyen, "A new combination of 1D and 2D filter banks for effective multiresolution image representation," 2008 15th IEEE International Conference on Image Processing, San Diego, CA, 2008, pp. 2820-2823.

[21] Vetterli M. and Kovacevé J., "Wavelets and Subband Coding," Upper Saddle River, NJ: Prentice-Hall, 1995.

[22] M. Feilner, D. Van De Ville and M. Unser, "An orthogonal family of quincunx wavelets with continuously adjustable order," in IEEE Transactions on Image Processing, vol. 14, no. 4, pp. 499-510, April 2005.

[23] Y Chen, M. D. Adams. and Wu-Sheng Lu, "Design of Optimal Quincunx Filter Banks for Image Coding," EURASIP Journal on Advances in Signal Processing, 2007.

[24] Beladgham, Moh, Bessaid, A, Taleb-Ahmed, Abdelmalik et Boukli Hacene, Ismail, "Medical image compression using quincunx wavelets and SPIHT coding," Journal of Electrical Engineering and Technology. vol. 7. no. 2, pp. 264-272, March 2012.

[25] A. Said. and W. Pearlman, "Set partition coding: part I of set partition coding and image wavelet coding systems," Foundations and Trend in Signal Processing, vol. 2, no.2, pp. 95-180, 2008.

[26] E, Christophe, a thesis on, "Compression d'image hypersperctrales et son impact sur la qulité des donées," Computer and Telecommunications Specialty: Signal and image, University of Toulouse, France, 2006. 
[27] Zhou Wang, A. C. Bovik, H. R. Sheikh and E. P. Simoncelli, "Image quality assessment: from error visibility to structural similarity," in IEEE Transactions on Image Processing, vol. 13, no. 4, pp. 600-612, April 2004.

[28] www.GE Medical System.com (database)

\section{BIOGRAPHIES OF AUTHORS}

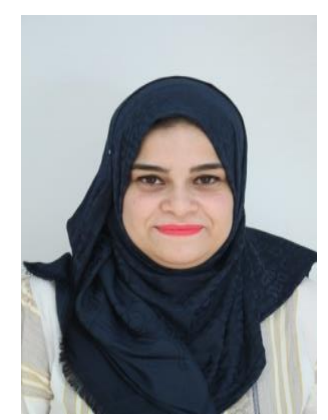

Imane Haouam, was born in Bechar (Algeria), received bachelor in 2011, and engineers degree in telecommunication from National Institute of Telecommunications and Information and Communication Technologies of Oran, Algeria, then master degree in networks and advanced telecommunication system from National Institute of Telecommunications and Information and Communication Technologies of Oran, Algeria, in 2016. His research interest includes image processiong, medical image compression, wavelet transform, active contour and image segmentation.

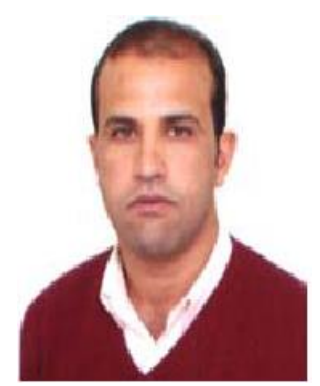

Mohammed Beladgham, was born in Tlemcen, Algeria; he received the electrical engineering diploma from university of Tlemcen, Algeria, and then a Magister in signals and systems from University of Tlemcen, Algeria and the PhD. degree in Electronics from the University of Tlemcen (Algeria), in 2012. His research interests are Image processing, Medical image compression, wavelets transform and optimal encoder. Correspondence address: Bechar University, Department of Electrical Engineering, Bechar, Algeria.

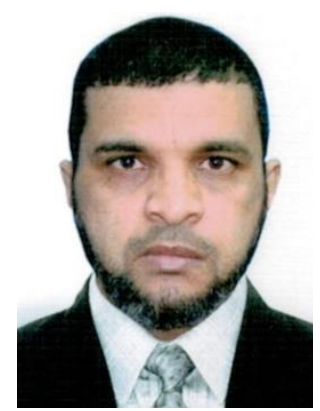

Ahmed Bouida, was born in Bechar (Algeria), received Bachelor in 1989 and Engineers degree in Electrical Engineering from the University Djillali Liabes of SidiBel Abbes (Algeria) in 1994 and his Magister degree in Electronic from the University Djillali Liabes of SidiBel Abbes (Algeria) in 1998 (Algeria). His current research interest includes Image processing, Biometric image compression, Second generation wavelets transform and Image quality assessment. 